\title{
1 What young people tell us about health-related social media and why we should listen
}

\author{
Victoria A. Goodyear and Kathleen M. Armour
}

\section{Chapter overview}

The pervasiveness of social media in young people's lives is widely acknowledged; yet, there is little robust evidence on the impacts of social media on young people's health and wellbeing. In this chapter, we explain the innovative research we have undertaken to understand, from young people's perspectives, the health-related issues and opportunities of social media. We explain key terms, including the new 'content-led pedagogical framework' and the 'pedagogical case model'. These tools were used to present, analyse, explain, and translate empirically rich data on young people's experiences of social media, and from stakeholder and academic groups from a range of disciplines.

\section{The focus of the book}

For many of the young people that we teach, coach, research, care for, parent, and support, it is important to remember that digital technology is regarded as an extension of self and social media is a primary mode of communication and social engagement. If, as adults, we want to reach these young people, understand something of their worlds, and offer support, we need to know how they engage with social media, what they learn from it and how that may influence their behaviours (Goodyear et al. 2018a, 2018b). While many influences may be positive, there are also likely to be periods of vulnerability in young people's lives where the sheer scale, intensity, and pervasiveness of social media could act to intensify those vulnerabilities. Social media is certainly a very powerful and dynamic feature of contemporary youth culture and, as such, it is important to understand how it operates in key areas of young people's lives.

This book adopts a novel approach to understanding, explaining, and communicating young people's experiences of health-related social media, and the impacts they report on their health, wellbeing, and levels of physical activity. The chapters are underpinned by robust data. Using empirically rich composite narrative case studies and evidence from multi-sector and multi-disciplinary stakeholders and academics, the book identifies the opportunities and risk-related impacts of social media for young people's health and wellbeing. It offers new 


\section{Victoria A. Goodyear and Kathleen M. Armour}

theoretical insights, as well as evidence-based and practical guidelines for relevant stakeholders including policy makers, schools, and health and education professionals/practitioners. The evidence presented in this book also provides information that will be important for parents/guardians and will help them to better understand how to engage with and respond to young people's contemporary needs.

The significance of this book resides in the insights it offers to address growing concerns around the world about young people's health and wellbeing (Inchley et al. 2017; Patton et al. 2016), and reported associations between young people's uses of social media and negative physical and mental health outcomes (Frith 2017; Swist et al. 2015; Third et al. 2017). Yet, there is limited robust evidence that explains whether and how social media influences young people's health-related knowledge and behaviours (Przybylski and Weinstein 2017a, 2017b). As a result, many adults are uncertain about how to support young people's engagement with health-related social media (Shaw et al. 2015; Third et al. 2017) and there is little guidance available from research and policy (Third et al. 2017; Wartella et al. 2016). This leaves adults ill-equipped both to protect young people from the negative influences of social media and to optimise the potential of social media as a medium for health promotion. This book, therefore, addresses a persistent societal question in new ways, and provides important evidence-based insights that are relevant to policy makers, researchers, health and education practitioners/professionals, and parents/ guardians who have an interest in supporting young people's health-related understandings and behaviours.

The book is organised into three main parts, and each can be read independently or in any order. In Part I, a series of data-rich case studies illustrates some of the many ways in which young people engage with social media and how and why this can have an influence on their health-related knowledge, understandings, and behaviours. In Part II, we step back from the vivid data and draw on a range of different disciplinary perspectives to better understand the ways in which health-related social media can influence young people. In Part III, the information from the previous sections is crystallised into evidence-based actions and guidelines that can help relevant adults to mitigate against risks while simultaneously maximising the positive and powerful potential of engagement with digital health-related media.

\section{The importance of new research on social media that listens to young people}

It has been reported from numerous international and socio-economic contexts that young people have the highest rates of social media use of any age group, and that they spend significant proportions of their time 'on' social media (Royal Society of Public Health [RPSH] 2017; Third et al. 2017). Turkle (2017) used the concept of 'tethered' to describe young people's prolific uses of social media and to highlight that young people want to be continuously 'connected'. Others 
have argued that young people's extensive and habitual uses of social media challenge the outdated notion that a dualism exists between 'real' life and online spaces (boyd 2014; Ito et al. 2010; Third et al. 2014). A dissolution of the online/offline binary is made apparent where social media operates as an active digital space for young people where relationships, identities, and intimacies are formed (boyd 2014; Handyside and Ringrose 2017; MacIsaac et al. 2018), and where learning can occur as a result of observing and communicating with peers of the same age (Ito et al. 2010). In this sense, social media is not merely a space where young people go to document their lives (Handyside and Ringrose 2017). Social media is a connected space for young people where communication, friendship, play, self-expression, and learning occur (Handyside and Ringrose 2017, MacIsaac et al. 2018).

There can be little doubt that understanding how social media influences young people's knowledge and behaviour is highly complex and difficult to navigate. There are diverse modes of social media (e.g. Snapchat or Instagram) that include varied and multi-dimensional interactive functionalities (e.g. likes or followers) (Highfield and Leaver 2016). The content created and accessed on social media is also user-generated, and shared in spaces where commercial, government, community, and individual contexts overlap (Freishtat and Sandlin 2010). In turn, social media disrupts the flow of traditional forms of health knowledge, where established learning and pedagogical concepts, procedures, and frameworks are problematic to apply in this highly interactive and dynamic context (Andersson and Öhman 2017; Andersson and Olson 2014; Goodyear 2017; Goodyear et al. 2018a). Understanding how social media influences knowledge and behaviour is even more challenging given that this medium is in a constant flux of change. New platforms, functions, and features are frequently introduced and adopted in youth culture (Miller et al. 2016), such as the rapid uptake of Snapchat in recent years that has, in turn, presented new issues related to temporality and memory (Handyside and Ringrose 2017; Highfield and Leaver 2016). Social media is, therefore, a very contemporary, dynamic, and interactive medium that engages young people. Navigating this type of media and understanding how the diverse, multi-user, and multi-functional spaces influence young people's knowledge and understanding is methodologically, theoretically, and ethically challenging.

To date, understandings of the health-related risks and opportunities of social media have been undermined by methodological weakness (Gaplin and Taylor 2017). Most studies fail to reflect the social complexity of the medium and/or the diverse ways in which young people navigate it. Evidence has been limited to one-off, short-duration intervention studies, analysis of parent/guardian and teacher perspectives, and/or evidence from survey data or observational methods (James 2014; Mascheroni, Jorge, and Farrugia 2014; Wartella et al. 2016). From these studies, health-related impacts of digital media engagement have been associated with time spent on social media, the platform, and/or the dissemination/accessibility of information (RPSH 2017; Shaw et al. 2015). Yet, the dynamic ways in which young people interact through social media (see boyd 
2014), and the powerful role of, for example, peers (Ito et al. 2010), likes (Jong and Drummond 2016), followers (MacIsaac et al. 2018), and selfies (Walsh 2017) are rarely considered. To understand how social media influences knowledge and behaviours, research must therefore account for the diverse multi-user and multi-functional interactive spaces of social media, and seek to better understand how young people use, navigate, and orientate themselves to these spaces.

The pressing need for new evidence on the dynamic and interactive ways in which young people engage with health-related social media is further indicated by the clear gaps that exist between adults and young people's understandings, where these gaps have been persistent (Buckingham 2016). International evidence suggests that young people value the accessibility of information from social media, and that they are increasingly turning to social media for health-related information (Swist et al. 2015; Third et al. 2017; Wartella et al. 2016). In existing research, young people have also reported on the benefits of social media in areas ranging from learning, socialisation, greater levels of social and emotional support, and creativity (Frith 2017; Swist et al. 2015; Third et al. 2017). Public discourse, however, tends to almost exclusively focus on social media and risk (boyd 2014). Adults also tend to assume that access to health information through social media will have negative knowledge-transmission effects that will impact on all young people in the same way (Third et al. 2017, 2014). Due to these perceived risks, there is a tendency to adopt protection-orientated approaches that seek to limit and control young people's social media use (boyd 2014; Livingstone, Mascheroni, and Staksrud 2018). As a result, many adults are unaware of the potential for social media to act as a positive health promotion resource and they fail to appreciate the opportunities that could stem from the dynamic and interactive ways in which young people use and navigate social media.

Despite young people's prolific engagement with social media, we, like others (Buckingham 2016; Hopkins 2010), are cautious of referring to the current generation as digital 'natives' or a digital youth generation. Nonetheless, we suggest that young people's very specific levels and forms of expertise in social media use should be recognised and accommodated. It is clear that young people are avid users and drivers of this contemporary, participatory, and user-driven online culture and, to this extent, they can be understood as highly skilled and knowledgeable. Understanding the ways in which young people use social media as a space for communication, entertainment, and learning could certainly challenge the social and cultural norms and expectations of adults (Ito et al. 2010; Livingstone et al. 2018). In the context of physical activity and health, for example, it has long been argued that understanding young people's perspectives is a powerful mechanism for designing new and more effective health and education interventions (Oliver and Kirk 2016; Leahy et al. 2016).

To understand how to better support young people's engagement with healthrelated social media, we argue that there is a need to learn from the experiences of young people. Any new guidelines or proposed interventions must chime with young people's needs and the ways in which they engage with social media. Developmentally, we know that adolescence is characterised by dynamic brain 
development and that interaction with the social environment shapes the capabilities an individual takes forward into adult life (Patton et al. 2016). In this context, social media can be a powerful social environment that can influence young people's current and future health-related behaviours. We also know that during adolescence, young people's social, emotional, and physical needs can change very rapidly, and this reinforces the need for relevant adults to be better informed about social media in order to offer appropriate support at particular points in time when young people might suddenly become vulnerable.

This book, therefore, addresses empirical, methodological, and theoretical gaps in our understandings about young people's engagement with health-related social media, and identifies new directions for research, policy, and practice. The aims of the book are to: (i) increase awareness of the opportunities and riskrelated impacts of social media on young people's health; (ii) generate new theoretical insights into young people's digital health and related behaviours; and (iii) inform new guidelines and actions for health and education practitioners and other relevant adults who have a role in supporting young people to engage with health-related social media in positive ways. In this chapter, we set the context for the book by: (1) providing an overview of the underpinning research project that generated the data for the case study chapters; (2) reviewing existing research and theory in the area of social media and young people; (3) proposing a refined understanding of the concept of pedagogy to better account for the findings from our research and that of others; and (4) providing a guide to the structure and organisation of the book and each chapter.

\section{New research: how we generated the data for the case study chapters}

The underpinning research for this book was undertaken at the University of Birmingham (UK) and was supported by the Wellcome Trust. ${ }^{1}$ Focusing on the key content areas of physical activity, diet/nutrition, and body image, we - Goodyear and Armour - sought to better understand from young people's perspectives, the health-related opportunities and issues generated by social media engagement. Between 2016-2017 we worked with a total of 1,346 young people and 35 key stakeholders working in areas related to young people's health and wellbeing. The project took place in three overarching phases.

Phase one of the research involved data collection in schools with young people. The aim was to generate new evidence on the types of health-related content young people access from social media, and to identify the types of content that they report as having an impact on their health-related knowledge and behaviours. Data collection involved 1,296 young people (age 13-18) from ten UK schools. The schools were located in diverse socio-demographic areas and the research included students from a range of ethnic backgrounds. A culturally responsive relational and reflexive approach to ethics was adopted (Sparkes and Smith 2014). This approach meant that ethical decision-making was guided by young people's needs and an awareness, understanding, and respect for their digital cultures (Goodyear 2017). Following this approach, data collection 
methods ensured participant safety, privacy, dignity, and autonomy. Informed consent and assent were provided, and legal conditions of social media were followed. Ethical approval was provided by The University of Birmingham Ethical Committee in July 2016.

In phase one, the data collection methods were initially co-constructed and then piloted with a group of ten young people (age 16-18), to ensure that the young people were able to communicate their experiences of social media. Participatory activities then took place in 12 different classes of young people across the ten schools $(n=236$, age $13-15, \mathrm{~m}=101, \mathrm{f}=135)$. The class activities involved the young people working in small groups to complete a series of tasks on an iBook and included: watching a video, completing a leaflet, and editing and creating a Pinterest digital pinboard. Using the data generated from the class activities, 19 focus group interviews $(n=84$, age $13-15, \mathrm{~m}=35, \mathrm{f}=49)$ took place with groups of young people from the class activities. Based on the data generated from the class activities and the interviews, an online survey was designed to 'test' the data in a wider sample of 1,016 young people within the same ten schools (age 13-16, $\mathrm{m}=334, \mathrm{f}=676$ ). The data from class activities, interviews, and the survey were then analysed using an adapted public pedagogies framework (explained in further detail below). The five themes that were constructed from the analysis process are reported in Chapters 2-7 and in the form of case study narratives. Further details on the methods and the analysis process can be found in Goodyear et al. (2018a).

Phase two of the research comprised of a workshop with 35 key stakeholders who have a responsibility for young people's health and wellbeing. The aim was to develop new guidelines and actions to help policy makers, researchers, health and education practitioners/professionals, and parents/guardians to support young people's engagement with health-related social media. Prior to the workshop, the text-based case studies (Chapters 2-7) were developed into short, 2-3minute digital animated case study videos. Grounded in the knowledge-to-action knowledge translation framework (Graham et al. 2006) the videos were created as knowledge translation tools to: (i) present the case study data in a clear, concise, user-friendly, and accessible format; and (ii) help stakeholders to identify, select, and create appropriate guidance and actions. The data-rich videos can be accessed here:

\section{http://epapers.bham.ac.uk/view/subjects/RC1200.html\#group_G}

The videos were presented to the stakeholders during the workshop and they were asked to provide an analysis of the risks and opportunities from their perspectives and to identify key actions. The profile of the stakeholder group was international, multi-sector, and multi-disciplinary and included teachers, international academics (UK, Ireland, Sweden, Netherlands, Spain, Australia, China), and trusts/organisations in the UK (such as the National Health service (NHS), Youth Sport Trust) that have a focus on youth health and wellbeing. The diversity of this stakeholder group resulted in insights into the case study data that 
were invaluable in seeking to reflect the diverse needs of young people and relevant adults. The stakeholder responses to the digital animated case study videos are reported in Chapters 2-7. In Chapters 8-15, a further theoretical analysis of the case studies is provided by some of the stakeholders who attended the workshop.

Phase three involved a workshop with 50 young people (age 13-16) from the ten schools that were involved in the data collection activities during phase one. Further grounded in the knowledge-to-action knowledge translation framework (Graham et al. 2006), the aims of this phase were to: (i) understand from the perspective of young people whether the guidelines and actions created by the stakeholders (phase two) were appropriate and would be effective; and (ii) identify the forms of support that young people perceive they require from adults. During the workshop, the young people watched the digital animated case study videos (phase two) and were presented with the responses of the stakeholders. In small groups, the young people then created a response for adults using artefacts explaining how young people their age should be supported to use social media for their health and wellbeing. The young people created and presented their response in different formats: radio or TV interviews, podcasts, movies, and newspaper articles. The data from the workshop were transcribed and then analysed using inductive and comparative techniques. The findings on how young people perceive adults should provide support is reported in Chapter 7. In the final step of the research, the data generated from the stakeholder (phase two) and young people (phase three) workshops were combined and further analysed. This analysis resulted in the development of evidencebased guidelines and actions that take into account stakeholder and young people's perspectives. These guidelines and actions can be accessed from Goodyear et al. (2018b).

The multi-layered, co-constructed, and participatory methods that were used in the project that underpins this book have ensured that the chapters are datarich and evidence-based. In the next section, we summarise the broader evidence-base in which the project is located. This provided the grounding for our focus on listening to young people, and the methodological and conceptual approaches that we adopted.

\section{Existing research: what can we learn from a review of the research on young people and social media}

Pioneering work in the study of young people and social media has been undertaken mainly in the fields of anthropology, psychology, and sociology. The research in these disciplines has provided important insights into the opportunities presented by social media. Research led, for example, by boyd, Ito, Livingstone, James, and Ringrose has identified opportunities for informal learning, identify formation, media/digital literacy, and understanding ethics and feminism/gender. This research provides an appropriate foundation for understanding how social media might influence young people's health-related 
knowledge and behaviours, and identifying risks and opportunities, as well as theoretical and conceptual orientations that might develop new understandings.

boyd $(2014,2007)$ is a notable pioneer in defining and characterising young people's participation in social media. Grounded in anthropology, boyd (2007) used the concept of networked publics to describe how young people's participation in social media is interconnected to the notion of an audience. boyd (2014, p. 8) argued that networked publics are: 'simultaneously (1) the space constructed through networked technologies and (2) the imagined community that emerges as a result of the intersection of people, technology and practice'. boyd $(2014,2007)$ claimed that networked publics have four main affordances that alter and amplify social dynamics: persistence, visibility, spreadability, and searchability (boyd 2014, 2007). While these affordances present new opportunities for young people's development by extending their capabilities to engage with established practices - such as socialisation, self-expression, and the sharing of information - many young people use social media to attract attention and increase visibility (boyd 2014). Although some adults find this behaviour concerning and even alarming, boyd (2014) argued that these 'teen' behaviours on social media are no different to those of a pre-digital age. As a result, boyd (2014) argued that networked publics are significant to the needs of young people and, for some, offer a lifeline to engage in socialisation activities (boyd 2014).

Similar to boyd (2007), Ito et al. (2010, p. 19) used the term networked publics to 'foreground the active participation of a distributed social network in the production and circulation of culture and knowledge'. In their anthropological work with young people, Ito et al. (2010) noted that networked publics created a powerful context for peer-based learning and they proposed that learning occurs in two types of networked publics: friendship-driven and interestdriven. Friendship-driven publics are spaces for social and emotional support, where young people engage in self-expression and both evaluate and give feedback to one another. Interest-driven publics are more specialised and niche, and are centred on a common topic. Interest-driven publics influence learning through young people's interactions with others who have greater expertise and/ or where young people can mentor others while simultaneously developing their leadership skills. The work of Ito et al. (2010), therefore, suggests that young people's health-related learning could be enhanced through the increased opportunities for socialisation that are provided by social media and through peerbased and interest-driven networks.

The importance of digital media for young people's learning, wellbeing and socialisation is reflected in the extensive body of work led by Livingstone (see Livingstone et al. 2018). From a social psychology perspective, Livingstone has explored how the changing conditions of mediation reshape everyday practices and present new risks and opportunities for young people's wellbeing. In terms of risks, Livingstone reported that young people are exposed to a significant amount of harmful, user-generated content, including in the areas of health. Examples include pro-anorexic or self-harm content (Livingstone et al. 2014). 
Yet, Livingstone et al. (2018) also reported that the risks for internet-using-young people are relatively low and exposure to content does not necessarily lead to harm. Based on these understandings, Livingstone, as well as others (see Third et al. 2014, 2017), have argued for digital access to be conceptualised as a fundamental 'right' for young people (Livingstone and Third 2017). From this rightsbased perspective, Livingstone et al. (2018) called for a focus on media literacy which involves empowering young people to engage with society through the media. By engaging with the media, Livingstone (2017) argues that young people will develop the skills and knowledge they need to engage effectively with social media, and the skills to determine what information is useful, misleading, trusted, and/or stems from political or commercial interests (Livingstone 2017). Fundamental to Livingstone's (2017) argument is that the more media literacy skills young people gain, the more opportunities they will be afforded.

While the works of boyd, Ito et al. and Livingstone suggest that that social media engagement can lead to positive impacts on young people's health-related knowledge and behaviours, there are a number of more pessimistic viewpoints. Turkle (2017), for example, has outlined troubling consequences related to attention, thinking, identities, and relationships. In particular, Turkle (2017) claimed that young people's addiction to social media can result in social alienation, hinder the ability to engage in interpersonal communications, and lead to the development of shallow relationships focused on self-expression. This view is further supported by Gardner and Davis (2014), who argued that young people's dependency on social media apps could undermine the development of healthy identities and relationships, and the capacity to think creatively.

The work of James and colleagues highlights the potentially problematic moral and ethical consequences resulting from young people's engagement with digital media (James 2014; James et al. 2014). Drawing on psychological models of moral development (see James et al., 2014), James (2014) outlined three ways in which young people approach digital media: self-focused, that involves a young person engaging with online activities where they mainly think about themselves; moral-focused, where a young person considers the consequences of online behaviour for other people they know; and ethically focused, where a young person considers the consequences of their behaviour for unknown individuals in wider social networks. The problematic issue for James (2014, p. 7) was that young people were 'principally, if not exclusively, concerned with their own interests when making decisions online'. This self-focused behaviour was reported, in some cases, to result in harm to a young person's peers and/or other individuals who were unknown to the young person. In this sense, while Livingstone (2017) suggests that media literacy will support young people's capacity to think critically about the digital information they encounter, James' (2014) research concludes that young people's understanding about how their individual behaviour impacts on others should also be addressed.

The importance of understanding how young people behave in their engagement with social media is further reflected in the research on gender and sexuality. Similar to James (2014), a number of scholars have pointed out that 
self-presentation on social media is of central importance to young people, and can drive the ways in which they participate, interact, and communicate (Handyside and Ringrose 2017; MacIsaac et al. 2018; Mascheroni, Vincent, and Jimenez 2015; Ringrose et al. 2013; Walsh 2017). It has also been reported that many young people use social media in a sexualised way, posting photos of their bodies that conform to normative sexualised stereotypes (Handyside and Ringrose 2017; Mascheroni, Vincent, and Jimenez 2015; Ringrose et al. 2013; Walsh 2017). While this content can be harmful by, for example, circulating images that have an affective influence on how others feel they should look and be (Ringrose et al. 2017), this self-focused behaviour also invites personal judgement, ridicule, and criticism (Handyside and Ringrose 2017; Mascheroni, Vincent, and Jimenez 2015). Furthermore, the images young people share of themselves and their bodies while, perhaps posted transiently, can take on an unanticipated 'life' (Handyside and Ringrose 2017). Similar to boyd's (2014) concept of visibility, Ringrose et al. (2013) reported that 'teen'-produced content can circulate widely and be used within peer networks as a form of digital currency. In turn, young people can become obsessed with the ways they look on social media, and it has been suggested that some are addicted to the feedback they obtain on whether their bodies conform to socially acceptable standards (Handyside and Ringrose 2017; Mascheroni, Vincent, and Jimenez 2015). In contrast to the more positive perspective of Ito et al. (2010) on the value of peerbased feedback, it is clear that research on gender and sexuality highlights some of the negative consequences of peer social media networks.

This brief overview has demonstrated that research from the fields of anthropology, psychology, and sociology has played an important part in advancing our understandings of the dynamic and interactive ways in which young people participate in social media. This work also emphasises the importance of understanding the issues and opportunities of digital media from young people's perspectives, and how participatory and ethnographic methods can generate new insights into learning, identity, media/digital literacy, ethics, and gender/sexuality. What is lacking from this perspective, however, is a specific focus on health and the ways in which young people's dynamic and interactive uses of social media shape their health-related knowledge and behaviours. Aside from Ito et al. (2010) there has also been a limited focus on learning, and there is a lack of understanding of the numerous factors that influence learning in a social media context. In the following section we consider the concept of pedagogy as a lens through which to interpret young people's health-related learning via social media. In addition to drawing upon existing understandings of pedagogy in health-related contexts, our research with young people and social media suggests that the concept of pedagogy could usefully be refined to fit the particular dynamism of social media. As a result, we propose some initial thoughts on the development of - what we have termed - a 'dynamic, content-led pedagogical framework'. 


\section{The case for reconsidering the concept of pedagogy in a social media context}

Pedagogy provides a conceptual lens to frame the ways in which learning occurs in varied and diverse contexts (Armour 2014). 'Sport and Exercise Pedagogy' is one variation on the concept of pedagogy as translated into the field of physical education, physical activity, and health (Armour 2014; Armour and Chambers 2014) and technology-mediated learning in physical activity and health contexts (Casey et al. 2017, 2016). According to Armour and Chambers (2014, p. 858), sport and exercise pedagogy focusses on:

(1) the needs of diverse learners of all ages in a wide range of physical activity/sport settings, including schools; (2) the abilities of professional teachers, coaches and instructors to meet those needs, and (3) the contexts in which relevant sport, exercise/health and physical education policy and knowledge are developed and delivered.

Sport and exercise pedagogy places young people's complex, diverse, and individual learning needs at its core (Armour 2014), and this focus aligns with the importance of understanding health-related social media from young people's perspectives. To date, however, the concept of sport and exercise pedagogy has been explored largely in formal contexts for learning, such as physical education or youth sport. It has also been argued that learning occurs at the moment where teaching/coaching practices, the curriculum, content/knowledge, and contexts align with and meet learners' needs (Armour 2014; Armour and Chambers 2014). Yet, in a social media context there is no defined teacher or curriculum and the contexts for learning are varied and shaped by multiple users and functionalities (Andersson and Olson 2014). As highlighted earlier in this chapter, social media is an informal context for learning and socialisation, where knowledge and behaviours can be influenced by diverse interactive functions (Walsh 2017), as well as by diverse people, who are either known (Ito et al. 2010) or unknown to individuals (James 2014). The concept of sport and exercise pedagogy is therefore limiting in framing learning within the dynamic and interactive context of social media.

Public pedagogies initially appeared to provide a neat umbrella concept to capture the informal learning context that characterises social media. Often grounded in the works of Giroux (2004), the concept of public pedagogies acknowledges learning as an experience that is influenced by popular culture (Burdick and Sandlin 2013). Giroux (2004) defined pedagogy as a political and moral practice, that represents the relationship between power, knowledge, and ideology (political) and how an individual invests in public life (moral). In turn, Giroux (2004) defined public pedagogy as the regulatory and emancipatory relationship between culture, power, and politics that occurs in a democratically configured social space. Giroux (2002) applied this concept of public pedagogy to explain how different forms of technology and media influence individuals' 
knowledge and behaviours, and this framework has been adopted widely in cultural and media studies (see Burdick and Sandlin 2013). For example, through particular representations in text, images, sounds, gestures, and dialogue, Giroux (2002, p. 539) argued that films operate pedagogically (i.e. politically and morally) through the "common sense assumptions that inform them, the affective investments they mobilise, as well as the absence and exclusions that limit the range of meanings and information available'.

Similar to our challenges with applying the definition of sport and exercise pedagogy to social media data, connecting Giroux's conceptualisation of public pedagogies to contemporary digital media is problematic. For us, as for others, it was challenging to apply Giroux's concept that was grounded in a passive media context to an interactive and dynamic social media context (Andersson and Olson 2014; Andersson and Öhman 2017; Reid 2010). In particular, an analytical framing of pedagogy that operates through traditional conceptualisations of power seemed to neglect the role of young people in shaping content and educational experiences (Andersson and Olson 2014) and that is evident in, for example, boyd (2014) and Ito et al.'s (2010) concept of networked publics.

Returning to some of the anthropological framings of young people's experiences with social media (i.e. boyd 2014; Ito et al. 2010), the recent digital anthropological work of Miller and colleagues (Miller et al. 2016) was helpful to us in framing how pedagogy functions in a social media context (Goodyear et al. 2018a). Miller et al.'s (2016) focus in social media research has been based on content. Content is significant to Miller et al. (2016) because it migrates through social media platforms and is an enduring entity in the dynamism of online/digital media. Content, however, is not passive. Miller et al. (2016) suggest that content can be conceptualised as something users do, whereby content is actively constructed through different cultural genres (i.e. styles of use).

In addition to digital anthropology, Lomborg's $(2011,2017)$ research derived from communication theories was also helpful in understanding pedagogy in a dynamic social media context. Lomborg (2017) applied the concept of cultural genres to social media research to interpret the development of knowledge through communication. Lomborg (2017) - as well as Miller et al. (2016) ground the concept of genres within the work of Goffman (1959), who argued that communication and sociality take place within cultural genres. In turn, Lomborg (2017) argued that genres provide analytical tools on the level of communicative practice. Genres are 'constituted by the interplay between interactive functionalities configured at the software level, and the invocation and appropriation of various software functionalities to achieve certain purposes, in and through users' actual communicative practices' (Lomborg 2017, n.p.).

By drawing on digital anthropology and communication theories, it was clear to us that an analytical framing of pedagogy in a social media context should be led by a focus on content. Content places the focus of analysis firmly on the active user and their interactions with content (Miller et al. 2016) and this focus 
aligned well with the research design that we adopted and that aimed to explain the data related to young people's perspectives (Goodyear et al. 2018a).

The data that we generated from young people about their engagement with health-related social media enabled us to pilot and develop the adapted conceptualisation of pedagogy (Goodyear et al. 2018a). The findings from this initial pilot work demonstrate that a content-led pedagogical framework can account for the very complex, dynamic, and interactive ways in which young people use social media, and can offer a framework to explain how young people's varying uses of social media influence their knowledge and behaviours (Goodyear et al. 2018a). Our content-led pedagogical framework, therefore, places content at its core (Figure 1.1) and can be understood as:

The interplay between the user and the interactive functionalities of digital media: (i) leading to the construction of content, and (ii) shaping how content influences knowledge and understandings (Figure 1).

(Goodyear et al. 2018a, p. 13)

The content-led pedagogical framework was used to analyse the data from the underpinning research, and construct five themes that are reported in the form of case study narratives in Chapters 2-6. This framework is still, however, in the very early stages of development. Although we are beginning to demonstrate that the framework can be used to explain digital engagement in different contexts - such as those related to health-related apps and online professional development courses - further development, application, and critical review is required. There is a need to ensure that a content-led pedagogical framework is robust, practical to use, and can meet the needs and demands of a range of researchers from different disciplines.

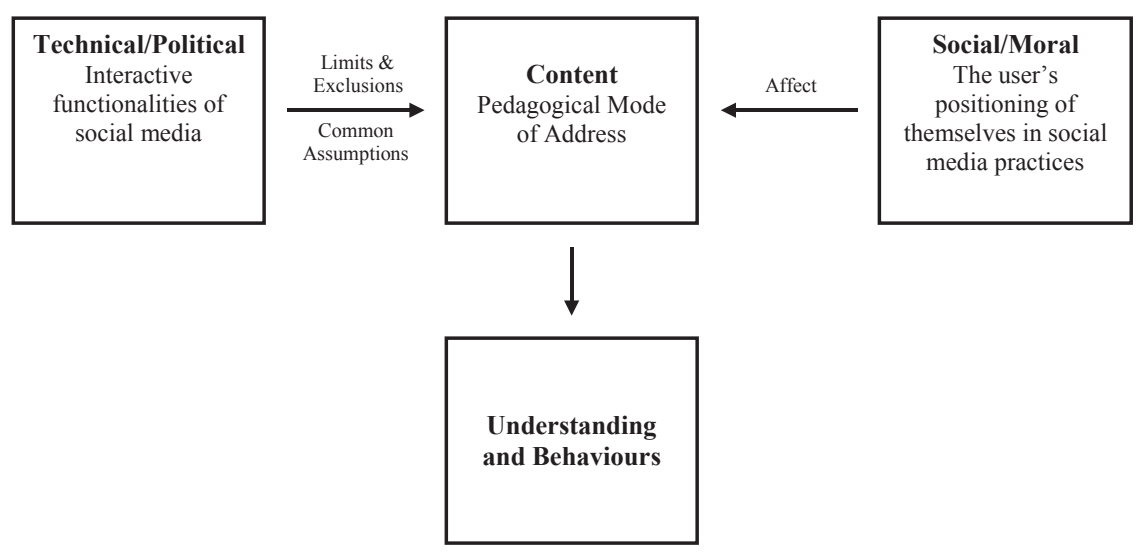

Figure 1.1 The operation of pedagogy in a social media context.

Source: Goodyear et al. 2018a, p. 13. 


\section{The structure and organisation of this book}

The central method for illustrating and interpreting the ways in which young people's understandings of health and wellbeing both construct and are constructed by social media is case study. As has been demonstrated in previous books by the editors (see Armour 2014, 2003; Armour and Jones 1998; Casey et al. 2016; Jones et al. 2003), case studies offer a powerful mechanism to: (i) portray the lives, practices, and experiences of individuals, and (ii) to connect theory to practice (see also Thomas 2011) in ways that add new insights. The 'Pedagogical Cases' model created by Armour (2014) sought to crystallise the potential of case studies as a bridge between persisting research/theory-practice gaps. Armour (2014) drew on the seminal work of Stenhouse (1980) who advocated for case studies to support practitioner learning in education. Stenhouse was keen to accumulate case studies that could bridge theory and practice by developing a shared language about practice. Yet, although case studies do feature in most branches of educational research, there has been little attempt to accumulate them in the ways in which Stenhouse envisaged. The aspirations to bridge research/theory practice effectively are also largely unmet, with Ball (2012) pressing for the development of new communication channels, translational mechanisms, and resources. The development of pedagogical cases was an attempt to develop such a mechanism, and to ensure that single discipline research could better support the complex needs of interdisciplinary practice.

In previous books that have used the pedagogical cases model, multidisciplinary teams of academics have analysed fictional narratives of individual young people (Armour 2014) and co-constructed teacher narratives (Casey et al. 2016). In this book, the pedagogical case study model has been refined further and the core narratives are constructed from rich empirical data on young people (see detail below). The data are represented in composite narratives of young people that illustrate the key findings of the research, and contained within each narrative are responses by key stakeholders. The theoretical analyses are presented in separate chapters, similar to the approach used in the ground-breaking work by Connell (1986). Taken together, the narratives in each of the pedagogical case models offer a useful starting point for developing a bank of cases around which stakeholder learning can be organised.

In this book, six evidence-based composite narrative case studies have been constructed from the editors' funded research projects ${ }^{2}$ undertaken with over 1,346 young people from the UK (as discussed in earlier sections of this chapter). What makes these case studies different from the previous pedagogical case books (see Armour 2014; Casey et al. 2016) is that each case study engages the voices of both young people and key stakeholders (e.g. schools, teachers, physical activity and health leaders in community settings, and policy and industry professionals). While case studies focused solely on young people (Armour 2014) and/or teachers and coaches (see Armour 2003; Armour and Jones 1998; Casey et al. 2016; Jones et al. 2003) provide rich narratives, they are limited in 
providing a 'complete picture' of physical activity and health education because the field crosses multiple settings. Social media is adding further complexity by increasing the diverse and easily accessible range of sources from which young people can learn about physical activity and health. It is interesting to note, for example, the absence of reference to physical education as a source of health education in the data from these young people (see Chapters 2-7).

In addition to composite case studies, this book follows the pedagogical cases model by offering insights into young people's uses of social media from diverse theoretical perspectives. As was noted earlier, theoretical analyses of young people and their engagement with social media have been undertaken predominantly in fields of anthropology, psychology, and sociology. Few connections between these theories have been made to date, and there have been very few theoretical insights into young people's uses of social media in the area of health and wellbeing. Given that social media has become an inherent part of young people's lives (boyd 2014; Turkle 2011), it is clear that this gap must be addressed, and this book represents an attempt to do just that. In Chapter 16, we summarise the contents of the book for multiple stakeholders and reflect on the effectiveness of the pedagogical case model in bridging the research-theorypractice divides, and the challenges of knowledge translation.

\section{Chapter contents}

In Chapters 2-7, composite narrative case studies of young people's engagement with health-related social media are presented. These chapters outline the different types of health-related content that young people access, create, and share on social media and that can influence their health-related knowledge and behaviours. The different types of content include:

- Automatically Sourced Content, that refers to the health-related information that social media sites pre-select and promote to young people (Kelly, Chapter 2).

- Suggested or Recommended Content, that refers to the process whereby young people's 'searches' for specific health-related information result in social media sites promoting vast amounts of partially related material to their accounts (Yaz, Chapter 3).

- Peer Content, that refers to the health-related content young people create, such as selfies (Leah, Chapter 4).

- Likes, that operate as a form of content through the process of affirmation (James, Chapter 5).

- Reputable Content, that refers to the power of specific social media accounts to frame the types of health-related information young people access and attend to (Jess, Chapter 6).

Chapter 7 presents a narrative case study of young people's recommendations and actions for adults. The chapter details how young people feel that different adults should support them to navigate health-related social media. 
Chapters $8-15$ provide a disciplinary analysis of the case study chapters from eight different disciplinary perspectives. Each of these chapters has a common format that includes: (i) a synthesis of existing research in the disciplinary area; (ii) case study analysis; and (iii) implications for supporting young people's engagement with health-related social media. These chapters focus on:

- A salutogenic perspective: outlining an alternative perspective on health that presents challenges to current policy, research and practice that is underpinned and often dominated by pathogenic perspectives. A salutogenic approach focuses on identifying new and diverse resources that young people draw on to support their health development. It is argued that social media can be a very powerful educative health resource (Quennerstedt, Chapter 8).

- School physical education: outlining how physical educators can contribute to young people's critical health literacy, develop pedagogies of affect, and deploy social media forms and content in critical and positive ways to support young people's health-related learning. This is an important contribution given the finding in the research that very few young people identified physical education as a source of health education (Kirk, Chapter 9).

- Disordered eating: outlines a continuum of problematic eating behaviours that ranges from unhealthy diets through to clinically diagnosed eating disorders, all of which can be influenced by content on social media. Valuable insights are provided into the important role of multi-component interventions to support young people's engagement with social media, including training for parents, teachers, school administrators, and local public health professionals, and where interventions are designed in collaboration with young people (Papathomas, White, and Plateau, Chapter 10).

- Space, place and identity: outlining the dualism between structure and agency in terms of how health is understood, portrayed, and performed in relation to social media. This is an important perspective that emphasises ways in which people's experiences of health-related social media are unique and varied based on differences in social landscapes (Sandford and Quarmby, Chapter 11).

- Public pedagogies: outlining how young people learn about their bodies, identities, and health through social media, and offering guidance as a counterbalance to the prevailing risk narrative. Recommendations are made on how to avoid technological determinism - that assumes social media is inherently oppressive or empowering - by focusing on embodied, affective learning in educational programmes (Rich, Chapter 12).

- Health literacy: outlining past and present public health agendas focused on developing patterns of health behaviours that transfer into adult life. This policy-informed perspective is vital to our understanding of young people and social media, and provides evidence of local and national interventions that can improve health literacy in response to social media content (Dudley, Van Bergen, McMaugh, and Mackenzie, Chapter 13). 
- Internet memes: outlines how content is created, shared, and mobilised through the internet, and the problematic consequences of algorithms in determining what young people see, act upon, and use from social media. These cultural-technological insights are central to our understandings of how health-related knowledge and behaviours are formed by social media engagement (Casey, Chapter 14).

- Digital democracy: outlining the importance of student voice in pedagogical innovation. This chapter offers critical insights into how risk-based narratives can be navigated in health and physical education contexts. Youth participatory action research is presented as an alternative way of working with young people to help young people and adults engage with and learn about technology integration (Enright and Gard, Chapter 15).

Chapters 16-17 outline the new directions for research, policy, and practice that are based on the evidence that has been generated and presented from young people, key stakeholders, and diverse academic disciplines. In Chapter 16 we Goodyear and Armour - evaluate whether the evidence, structure, and formatting of this book have been successful in providing policy makers, researchers, and health and education and practitioners/professionals with information that will help them to understand and better support young people's engagement with health-related social media. We do this by summarising the key findings for different stakeholder groups, and reflecting upon the value of the pedagogical cases framework and the challenges of engaging in successful knowledge translation and exchange. We also identify the further questions that the findings of this research raise. In Chapter 17 an independent analysis of the evidence and the book is provided by Cale. This final chapter outlines gaps in the existing evidence-base, limitations, and strengths of the book, and provides direction on the practices and/or interventions that will be effective in supporting, developing, and enhancing young people's health-related knowledge and behaviours.

\section{Notes}

1 The Wellcome Trust are a global charitable foundation, and are a UK-based research funding body. For further details, please see: https://wellcome.ac.uk/about-us

2 Details on the underpinning research funded by the Wellcome Trust can be accessed here: https:/wellcome.ac.uk/what-we-do/directories/seed-awards-humanities-social-sciencepeople-funded

\section{References}

Andersson, E. and Öhman, J., 2017. Young people's conversations about environmental and sustainability issues in social media. Environmental Education Research, 23, 465-485.

Andersson, E. and Olson, M., 2014. Political participation as public pedagogy - the educational situation in young people's political conversations in social media. Journal of Social Science Education, 13, 115-126. 
Armour, K.M., 2014. Pedagogical Cases in Physical Education and Youth Sport. London: Routledge.

Armour, K.M. and Chambers, F.C., 2014. 'Sport and exercise pedagogy'. The case for a new integrative sub-discipline in the field of sport and exercise sciences/kinesiology/ human movement sciences. Sport, Education and Society, 19, 855-868.

Armour, K.M. and Jones, R.L., 1998. Physical Education Teachers' Lives and Careers: PE, Sport and Educational Status. London: Routledge.

Ball, A.F., 2012. 2012 Presidential Address. To know is not enough: Knowledge, power and the zone of generativity. Educational Researcher, 41, 8, 283-293.

boyd, d., 2014. It's Complicated: The Social Lives of Networked Teens. London: Yale University Press.

boyd, d., 2007. Why youth (heart) social network sites: the role of networked publics in teenage social life. In: D. Buckingham, ed., MacArthur Foundation Series on Digital Learning - Youth, Identity, and Digital Media. Cambridge, MA: MIT Press, 119-142.

Buckingham, D., 2016. Is there a digital generation? In: D. Buckingham and R. Willett, eds. Digital Generations: Children, Young People and the New Media. London: Routledge, 1-18.

Burdick, J. and Sandlin, J.A., 2013. Learning, becoming, and the unknowable: Conceptualizations, mechanisms and processes in public pedagogy literature. Curriculum Inquiry, 43, 142-177.

Casey, A., Goodyear, V.A., and Armour, K.M., 2017. Rethinking the relationship between pedagogy, technology and learning in health and physical education. Sport, Education and Society, iFirst, 22(2), 288-304.

Casey, A., Goodyear, V.A., and Armour, K.M., 2016. Digital Technologies and Learning in Physical Education: Pedagogical Cases. London: Routledge.

Connell, R.W., 1986. Teachers' Work. London: George, Allen \& Unwin.

Frith, E., 2017. Social media and children's mental health: A review of the evidence. Available from: from: https://epi.org.uk/wp-content/uploads/2017/06/Social-Media Mental-Health_EPI-Report.pdf

Gaplin, A. and Taylor, G., 2017. Changing behaviour: Children, adolescents and screen use. Accessed from: www.bps.org.uk/sites/beta.bps.org.uk/files/Policy\%20-\%20Files/ Changing\%20behaviour\%20-\%20children $\% 2$ C\%20adolescents $\% 2 \mathrm{C} \% 20$ and $\% 20$ screen\%20use.pdf

Gardner, H. and Davis, K., 2014. The App Generation. New Haven, CT: Yale University Press.

Giroux, H.A., 2004. Public pedagogy and the politics of neo-liberalism: Making the political more pedagogical. Policy Futures in Education, 2, 494-503.

Giroux, H.A., 2002. From 'Manchild' to 'Baby Boy': Race and the politics of self-help. $J A C, 22,527-560$.

Goffman, E. 1959. The Presentation of the Self in Everyday Life. Garden City, NY: Anchor Books.

Goodyear, V.A., 2017. Social media, apps, and wearable technologies: Navigating ethical dilemmas and procedures. Qualitative Research in Sport, Exercise and Health, 9(3), 285-302.

Goodyear, V.A., Armour, K.M., and Wood, H., 2018a. Young people and their engagement with health-related social media: New perspectives. Sport, Education and Society, iFirst, doi:10.1080/13573322.2017.1423464.

Goodyear, V.A., Armour, K.M., and Wood, H., 2018b. The Impact of Social Media on Young People's Health and Wellbeing: Evidence, Guidelines and Actions. Birmingham, UK: University of Birmingham. 
Graham, I.D., Logan, J., Harrison, M.B., Straus, S.E., Tetroe, J., Caswell, W., and Robinson, N., 2006. Lost in knowledge translation: Time for a map? Journal of Continuing Education in the Health Professions, 26, 13-24.

Handyside, S. and Ringrose, J., 2017. Snapchat memory and youth digital sexual cultures: Mediated temporality, duration and affect. Journal of Gender Studies, 26(3), 347-360.

Haussmann, J.D., Touloumtzis, C., White, M.T., Colbert, M.D., and Golding, H.C., 2017. Adolescent and young adult use of social media for health and its implications. Journal of Adolescent Health, 60(6), 714-719.

Highfield, T. and Leaver, T., 2017. Instagrammatics and digital methods: Studying visual social media, from selfies and GIFs to memes and emoji. Communication Research and Practice, 2 (1), 47-62.

Holmberg, C., Chaplin, E.J., Hillman, T., and Berg, C., 2016. Adolescents' presentations of food in social media: An explorative study. Appetite, 99, 121-129.

Hopkins, P.E., 2010. Young People, Place and Identity. Abingdon, Oxon: Routledge.

Inchley, J., Currie, D., Jewell, J., Breda, J., and Barnekow, V., eds., 2017. Adolescent obesity and related behaviours: Trends and inequalities in the WHO European Region, 2002-2014. Observations from the Health Behaviour in School-aged Children (HBSC) WHO collaborative cross-national study. Copenhagen, WHO Regional Office for Europe.

Ito, M., Baumer, S., Bittanti, M., boyd, d., Cody, R., et al., 2010. Hanging Out, Messing Around, and Geeking Out: Kids Living and Learning with New Media. Cambridge, MA: MIT Press.

James, C., 2014. Disconnected: Youth, New Media and the Ethics Gap. London: MIT Press.

James, C., Davis, K., Flores, A., Francis, J.M., Pettingill, L., et al., 2014. Young People, Ethics, and the New Digital Media. London: MIT Press.

Jones, R.L., Armour, K.M., and Potrac, P., 2003. Sports Coaching Cultures: From Practice to Theory. London: Routledge.

Jong, S.T. and Drummond, M.J.N., 2016. Hurry up and 'like' me: Immediate feedback on social networking sites and the impact on adolescent girls. Asia-Pacific Journal of Health, Sport and Physical Education, 7(3), 251-267.

Leahy, D., Burrows, L., McCuaig, L., Wright, J., and Penney, D., 2016. School Health Education in Changing Times. London: Routledge.

Livingstone, S., 2017. Children's and Young People's Lives Online. In: D. Brown, ed., Online Risk to Children: Impact, Protection and Prevention. London: Wiley Blackwell, 23-36.

Livingstone, S., Mascheroni, G., Ólafasson, K., and Haddon, L., 2014. Children's Online Risks and Opportunities: Comparative Findings from EU Kids Online and Net Children Go Mobile. London: London School of Economics and Political Science.

Livingstone, S., Mascheroni, G., and Staksrud, R., 2017. European research on children's internet use: Assessing the past and anticipating the future. New Media \& Society, 20(3), 1103-1122.

Livingstone, S. and Third, A., 2017. Children and young people's rights in the digital age: An emerging agenda. New Media \& Society, 19(5), 657-670.

Lomborg, S., 2017. Social Media, Social Genres. London: Routledge.

Lomborg, S., 2011. Social media as communicative genres. Journal of Media and Communication Research, 51, 55-71.

MacIsaac, S., Kelly, J., and Gray, S., 2018. 'She has like 4000 followers!': The celebrification of self within school social networks. Journal of Youth Studies,21(6), 816-835. 
Mascheroni, G., Jorge, A., and Farrugia, L., 2014. Media representations and children's discourses on online risks: Findings from qualitative research in nine European countries. Cyberpsychology: Journal of Psychosocial Research in Cyberspace, 8(2), article 2 .

Mascheroni, G., Vincent, J., and Jimenez, E., 2015. 'Girls are addicted to likes so they post semi-naked selfies': Peer mediation, normativity and the construction of identity online. Cyberspychology: Journal of Psychosocial Research on Cyberspace, 9(1), article 5 .

Miller, D., Costa, E., Haynes, N., McDonald, T., Nicolescu, R., et al., 2016. How the World Changed Social Media. London: UCL Press.

Oliver, K.M. and Kirk, D., 2016. Towards an activist approach to research and advocacy for girls and physical education. Physical Education and Sport Pedagogy, 21, 313-327.

Patton, G.C., Sawyer, S.M., Santelli, J.S., Ross, D.A., Afifi, R. et al., 2016. Our future: A Lancet commission on adolescent health and wellbeing. Lancet, 387, 2423-2478.

Reid, A., 2010. Social media, public pedagogy and the end of private learning. In: J.A. Sandlin, B.D. Shultz, and J. Burdick, eds., Handbook of Public Pedagogy. London: Routledge, 194-200.

Ringrose, J., Harvey, L., Gill, R., and Livingstone, S., 2013. Teen girls, sexual double standards and 'sexting': Gendered value in digital image exchange. Feminist Theory, 14, 305-323.

Royal Society of Public Health., 2017. \#Status on Mind: Social Media and Young People's Mental Health and Wellbeing. London: Royal Society for Public Health.

Shaw, J.M. Mitchell, C.A., Welch, A.J., and Williamson, M.J., 2015. Social media used as a health intervention in adolescent health: A systematic review of the literature. Digital Health, 1, 1-10.

Stenhouse, L., 1980. Presidential Address: The study of samples and the study of cases. British Educational Research Journal, 6, 1, 1-6.

Sparkes, A. and Smith, B., 2014. Ethical issues in qualitative research. In: A. Sparkes and B. Smith, eds., Qualitative Research Methods in Sport, Exercise and Health: From Process to Product. London: Routledge, 206-237.

Swist, T., Collin, P., McCormack, J., and Third, A., 2015. Social Media and the Wellbeing of Children and Young People: A Literature Review. Perth, WA: Prepared for the Commissioner for Children and Young People, Western Australia.

Third, A., Bellerose, D., Oliveira, J.D.D., Lala, G., and Theakstone, G., 2017. Young and Online: Children's Perspectives on Life in the Digital Age. Sydney: Western Sydney University.

Third, A., Bellerose, D., Dawkins, U., Keltie, E., and Pihl, K., 2014. Children's Rights in the Digital Age: A Download from Children Around the World. Melbourne: Young and Well Cooperative Research Centre.

Thomas, G., 2011. How To Do Your Case Study. London, Sage.

Turkle S. 2017. Alone Together: Why We Expect More from Technology and Less from Each Other. 3rd edn. New York: Basic Books.

Walsh, J., 2017. Adolescents and Their Social Media Narratives. London: Routledge.

Wartella, E., Rideout., V., Montague, H., Beaudoin-Ryan, L., and Lauricella, A., 2016. Teens, health, and technology: A national survey. Media and Communications, 4(3), $12-23$. 